\title{
Estudio del proceso de sinterización reactiva en sistemas con dolomita mediante termodifractometría de neutrones
}

\author{
A. H. DE AZA', X. TURRILlAS ${ }^{\sharp, \Theta}$, J. L. RODRÍGUEZ; P. PENA \\ *Instituto de Cerámica y Vidrio (CSIC). Campus de Cantoblanco. Cantoblanco (Madrid). España. \\ * Instituto de Ciencias de la Construcción Eduardo Torroja (CSIC). Madrid (España). \\ ${ }^{\theta}$ European Synchrotron Radiation Facility (ESRF). Grenoble. Francia. \\ - CINVESTAV-IPN. Saltillo (Coahuila). México.
}

\begin{abstract}
La utilización de la difracción de neutrones a alta temperatura, aplicada a la investigación de procesos de sinterización reactiva, permite el estudio de las reacciones que tienen lugar in situ y, debido a su excelente poder de resolución temporal, la identificación de posibles fases transitorias de corta vida. Este trabajo recoge algunos de los resultados obtenidos durante la descomposición de la dolomita $\mathrm{MgCa}\left(\mathrm{CO}_{3}\right)_{2} \mathrm{y}$ los primeros estadios (hasta $1300^{\circ} \mathrm{C}$ ) de la sinterización reactiva de mezclas de dolomita con alúmina $\left(\mathrm{Al}_{2} \mathrm{O}_{3}\right)$ y dolomita con circón $(\mathrm{ZrSiO}$ ). Teniendo en cuenta estos datos se han establecido las secuencias de reacción, así como la formación de distintas fases transitorias en cada uno de los sistemas. Así mismo, se han determinado las energías de activación correspondientes a la descomposición del MgCa(CO $)_{2}$ y del $\mathrm{CaCO}_{3}$ en compactos de $\mathrm{MgCa}\left(\mathrm{CO}_{3}\right)_{2}$ puro y en las mezclas estudiadas. Adicionalmente, mediante refinamiento Rietveld se ha determinado la variación con la temperatura de los parámetros cristalinos a y $c$ de la celda unidad del $\mathrm{MgCa}\left(\mathrm{CO}_{3}\right)_{2}$ hasta temperaturas próximas a su descomposición $\left(700^{\circ} \mathrm{C}\right)$.
\end{abstract}

Palabras clave: Termodifractometría de neutrones. Dolomita, $\mathrm{MgCa}\left(\mathrm{CO}_{3}\right)_{2}$. Sinterización reactiva.

Study of reaction sintering in dolomite based systems by neutron termodifractometry.

Due to their adequate time-resolution, high temperature Powder Neutron Diffraction applied to reaction-sintering process allows in situ reaction analyses and transient phase identifications, which otherwise cannot be detected by quenching and conventional diffraction methods. Bearing in mind that dolomite $\mathrm{MgCa}\left(\mathrm{CO}_{3}\right)_{2}$ is often used as a raw material in the synthesis of different refractory materials, in the present work, neutron diffraction time-resolved experiments were carried out during $\mathrm{MgCa}\left(\mathrm{CO}_{3}\right)_{2}$ decomposition and the first steps of the reaction-sintering process of $\mathrm{MgCa}\left(\mathrm{CO}_{3}\right)_{2}-\mathrm{Al}_{2} \mathrm{O}_{3}$ and $\mathrm{MgCa}\left(\mathrm{CO}_{3}\right)_{2}-\mathrm{ZrSiO}$ mixtures. The results obtained up to $1300^{\circ} \mathrm{C}$ are presented and discussed to understand how these minerals behave during these first steps of the reaction sintering process. The activation energy for dolomite and calcite breakup was calculated from fitting to a contracting sphere model. Additionally, the dolomite mean thermal expansion coefficients were calculated along a and c axes from $25^{\circ}$ to $700^{\circ} \mathrm{C}$.

Key words: Neutron diffractometry. Dolomite. $\mathrm{MgCa}\left(\mathrm{CO}_{3}\right)_{2}$. Reaction sintering.

\section{INTRODUCCIÓN}

La utilización de dolomita $(\mathrm{MgCa}(\mathrm{CO}))$, materia prima natural de elevada pureza, es una alternativa económicamente rentable para la producción de diversos materiales refractarios estructurales. Se ha demostrado que partiendo de mezclas de dolomita con alúmina $(\mathrm{AlO})$ se pueden obtener nuevos cementos aluminosos con espínela $\left({ }^{2} \mathrm{MgAl} \mathrm{O}^{3}\right)$ para su uso en siderurgia ${ }^{1}$ y de su mezcla con circón $(\mathrm{ZrSiO}),{ }^{2}$ distintos materiales refractarios de magnesia conteniendo circonato cálcico ( $\mathrm{CaZrO}$ ) para su empleo, en hornos rotatorios de cemento ${ }^{2,3,4}$. Con la finalidåd de estudiar las reacciones que tienen lugar durante el proceso de producción de estos materiales, se han realizado series de experimentos en un horno de alta temperatura instalado en un equipo de difracción de neutrones ${ }^{5}$.

La utilización de la difracción de neutrones a alta temperatura, aplicada a la investigación de procesos de sinterización reactiva, permite el estudio in situ de las reacciones que tienen lugar y, gracias a su excelente poder de resolución temporal, la identificación de posibles fases transitorias de corta vida. Adicionalmente, se pueden realizar estudios cinéticos de dichos procesos. La realización de las medidas en modo de transmisión permite analizar la muestra en volumen y obtener información representativa del interior de muestras relativamente grandes, lo que proporciona una mejor estadística, y en el caso de carbonatos permite obtener datos representativos sobre los mecanis- mos de reacción imperantes a la presión parcial de $\mathrm{CO}_{2}$ existente en el interior de las muestras. Esta técnica permite obtener una información simultanea de los cambios que tienen lugar en la composición mineralógica y en las características estructurales de las fases presentes en el material durante el calentamiento.

Este trabajo recoge algunos de los resultados obtenidos durante la descomposición de la dolomita ${ }^{6}$ y de la calcita, formada tras la descomposición de esta, en compactos de dolomita pura y en mezclas de dolomita con alúmina o con circón. Así mismo se han determinado las energías de activación correspondientes a las citadas descomposiciones y se ha calculado la variación con la temperatura de los parámetros cristalinos $a$ y $c$ de la celda unidad del $\mathrm{MgCa}\left(\mathrm{CO}_{3}\right)_{2}$ hasta temperaturas próximas a su descomposición $\left(700^{\circ} \mathrm{C}\right)$. Además se muestran los estadios mas tempranos (hasta $1300^{\circ} \mathrm{C}$ ) de la sinterización reactiva de mezclas de dolomita-alúmina y dolomita-circón ${ }^{4,7}$.

\section{PROCEDIMIENTO EXPERIMENTAL}

Como materia prima se utilizó una dolomita natural, dolomita Micro $15^{\circledR}$, suministrada por la empresa Prodomasa, (Productos Dolomíticos de Málaga, S.A., Coín, Málaga). Se trata de una dolomita 


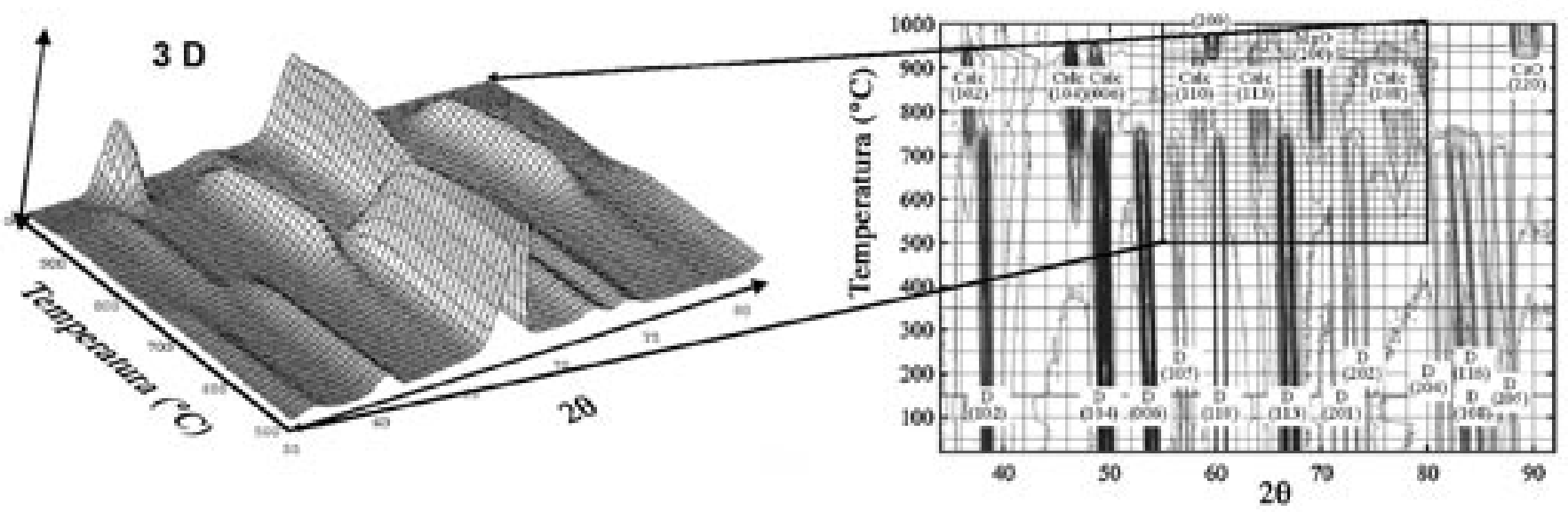

Figura 1. Secuencia de difractogramas registrada hasta $1000^{\circ} \mathrm{C}$ durante la descomposición de la dolomita en el equipo D20. (a) Representación tridimensional. (b) Proyección del diagrama tridimensional en una representación bidimensional. Se pueden observar los correspondientes índices de Miller asignados a cada fase. $\mathrm{D}$ denota dolomita, Calc calcita, $\mathrm{CaO}$ cal y $\mathrm{MgO}$ magnesia.

y desaparición de fases con mayor exactitud se procedió a proyectar el diagrama tridimensional en una representación bidimensional que se muestra en la Figura 1-b. A las distintas fases observadas se les ha asignado los correspondientes índices de Miller para su correcta identificación. De estas figuras se deduce, de una manera sencilla, que el proceso de descomposición del $\mathrm{MgCa}\left(\mathrm{CO}_{3}\right)_{2}$ comienza a temperaturas cercanas a $\sim 550^{\circ} \mathrm{C}$ en dos etapas sucesivas: a) descomposición del $\mathrm{MgCa}\left(\mathrm{CO}_{3}\right)_{2}$ generando $\mathrm{CaCO}_{3}$ y $\mathrm{MgO}$ y b) descomposición del $\mathrm{CaCO}_{3}$ con la consecuente formación de $\mathrm{CaO}$ a aproximadamente $950^{\circ} \mathrm{C}$.

En las mezclas de dolomita con alúmina o con circón, en las proporciones de las ecuaciones $\{1\}$ y $\{2\}$, se pudo registrar, según el caso, la secuencia de reacción de los productos de descomposición del $\mathrm{MgCa}\left(\mathrm{CO}_{3}\right)_{2}$ con el $\mathrm{Al}_{2} \mathrm{O}_{3}$ o con el $\mathrm{ZrSiO}_{4}$

En el primero de los casos se pudo observar la reacción de la alúmina con el $\mathrm{MgO}$ y con el $\mathrm{CaO}$ procedentes de la citada descomposición. Dando lugar a la formación de: $\mathrm{CaAl}_{2} \mathrm{O}_{4}\left(\sim 900^{\circ} \mathrm{C}\right), \mathrm{MgAl}_{2} \mathrm{O}_{4}$ (entre $\sim 1000^{\circ}-\sim 1100^{\circ} \mathrm{C}$ ) y $\mathrm{CaAl}_{4} \mathrm{O}_{7}$ (comenzando entorno a $\sim 1200^{\circ} \mathrm{C}$ ), coexistiendo estas tres fases en equilibrio al final del proceso. Esta secuencia de fases esta en concordancia con resultados previos obtenidos mediante otras técnicas mas convencionales ${ }^{1}$.

Por otro lado, en el segundo de los casos estudiados, el proceso de reacción de los productos de descomposición del $\mathrm{MgCa}\left(\mathrm{CO}_{3}\right)_{2}$ con el $\mathrm{ZrSiO}_{4}$ tiene lugar con la formación de fases transitorias tales como $\mathrm{ZrO}_{2}$-tetragonal $\left(\sim 775^{\circ}-1000^{\circ} \mathrm{C}\right), \mathrm{Ca}_{3} \mathrm{MgSi}_{2} \mathrm{O}_{8}\left(\sim 775^{\circ}-1100^{\circ} \mathrm{C}\right)$ y $\alpha-$ $\mathrm{Ca}_{2} \mathrm{SiO}_{4}\left(\mathrm{~T}>900^{\circ} \mathrm{C}\right)$, que conducen a la formación de las fases estables al final del proceso: $\mathrm{MgO}-\mathrm{CaZrO}_{3}-\mathrm{Ca}_{2} \mathrm{SiO}_{4}$. En la Figura 2, se muestra una proyección bidimensional de la secuencia de difractogramas registrada hasta $1300^{\circ} \mathrm{C}$ en el equipo D1B para la mezcla de dolomitacircón. A las distintas fases observadas se les ha asignado los correspondientes índices de Miller para su identificación. Una secuencia de las reacciones propuestas a lo largo del proceso se puede encontrar en un trabajo complementario.

En los dos casos estudiados la secuencia de formación de las distintas fases durante las reacciones esta de acuerdo con sus energías libres de formación, como se corroboró mediante cálculos termodiná$\operatorname{micos}^{1,4}$.

En las tres muestras estudiadas, la energía de activación de la descomposición de los carbonatos se calculó utilizando el procedimiento descrito, para procesos no isotermos, por Coats y Redfern ${ }^{9} \mathrm{y}$ aplicando el modelo cinético de contracción de la esfera propuesto 


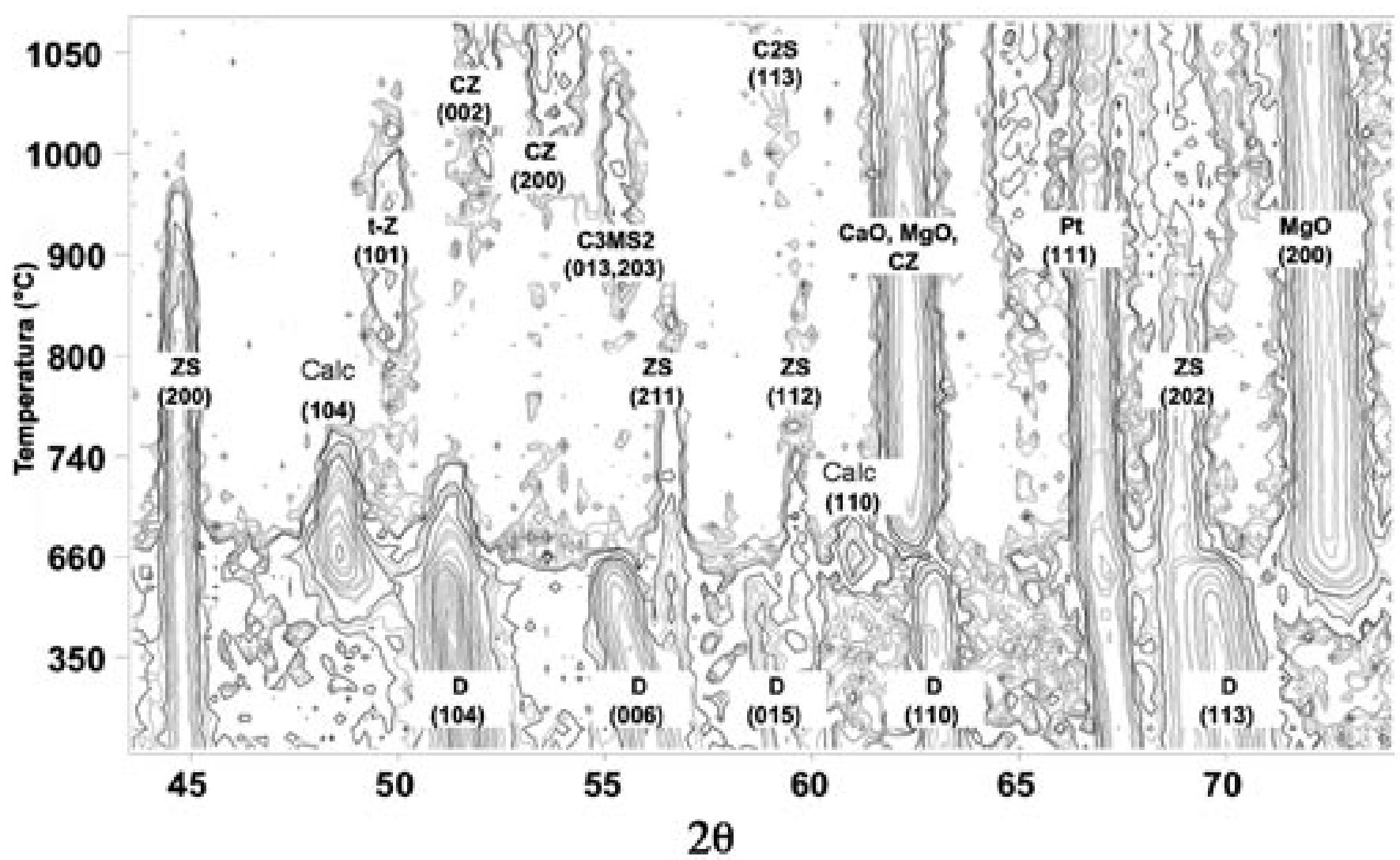

Figura 2. Proyección bidimensional de la secuencia de difractogramas registrada durante el proceso de reacción de los productos de descomposición del $\mathrm{MgCa}\left(\mathrm{CO}_{3}\right)_{2}$ con el $\mathrm{ZrSiO}_{4}$ hasta $1300^{\circ} \mathrm{C}$ en el equipo D1B. A las distintas fases observadas se les ha asignado los correspondientes índices de Miller para su identificación. Notación empleada: D por dolomita, Calc por calcita, $\mathrm{ZS}$ por circón, $\mathrm{C}_{3} \mathrm{MS}_{2}$ por merwinita, $\mathrm{C}_{2} \mathrm{~S}$ por $\alpha-\mathrm{Ca}_{2} \mathrm{SiO}_{4^{\prime}} \mathrm{CZ}$ por circonato cálcico y Pt por platino.

por Hume-Colvin ${ }^{10}$ para la descomposición de carbonatos, siguiendo la expresión:

$$
\left[1-(1-\alpha)^{1 / 3}\right]-2 \ln \mathrm{T}=\ln \mathrm{AR} / \mathrm{E}_{\mathrm{a}} \beta-\mathrm{E}_{\mathrm{a}} / \mathrm{RT}
$$

donde $\mathrm{T}$ es la temperatura absoluta en Kelvin, A es el factor preexponencial de Arrhenius, $\mathrm{R}$ la constante de los gases perfectos, $\beta$ es la velocidad de calentamiento, $\alpha$ indica el avance de la reacción y E es la energía de activación. Representando $\ln 3\left[1-(1-\alpha)^{1 / 3}\right]-2 \ln \mathrm{T}$ frente a $1 / \mathrm{T}$ se obtiene la energía de activación ( $\left.\mathrm{E}_{\mathrm{a}}\right)$ como resultado de la pendiente de dicha representación gráfica.

La evolución de las reacciones de descomposición del $\mathrm{MgCa}\left(\mathrm{CO}_{3}\right)_{2}$ y del $\mathrm{CaCO}_{3}$ se cuantificaron mediante la variación de las intensidades integradas (medida del área) de picos de difracción, adecuadamente seleccionados, en función de la temperatura. Con objeto de minimizar las interferencias originadas por el resto de las fases y el dispositivo experimental, se utilizo, en el presente caso, la reflexión (110) del $\mathrm{MgCa}\left(\mathrm{CO}_{3}\right)_{2}$ y del $\mathrm{CaCO}_{3}$. Se escogió esta reflexión ya que su intensidad se ve poco afectada por la agitación térmica de los átomos en la dirección del eje $c$. El progreso de las reacciones de descomposición se evaluó utilizando las intensidades integradas debidamente normalizadas a 1 . Así $\alpha=1$ indica que la reacción de descomposición del carbonato no ha empezado y $\alpha=0$ que el proceso ha terminado. El modo de proceder en este tipo de cálculos se muestra con todo tipo de detalles en las referencias ${ }^{6,9,10}$.

En la Tabla I se exponen los valores de energía de activación cal-
TABLA I. ENERGías DE ACTIVACIÓN CALCULADAS PARA LA DESCOMPOSICIÓN DEL $\mathrm{MgCa}\left(\mathrm{CO}_{3}\right)_{2}$ y el $\mathrm{CaCO}_{3}$.

\begin{tabular}{|c|c|c|}
\hline Muestra & $\begin{array}{c}\mathrm{E}_{\mathrm{a}}\left(\mathrm{MgCa}\left(\mathrm{CO}_{3}\right)_{2}\right) \\
\left(\mathrm{Kcal} \mathrm{mol}^{-1}\right)\end{array}$ & $\begin{array}{c}\mathrm{E}_{\mathrm{a}}\left(\mathrm{CaCO}_{3}\right) \\
\left(\mathrm{Kcal} \mathrm{mol}^{-1}\right)\end{array}$ \\
\hline $\mathrm{MgCa}\left(\mathrm{CO}_{3}\right)_{2}$ & 56 & $(-)$ \\
$\mathrm{MgCa}\left(\mathrm{CO}_{3}\right)_{2}-\mathrm{Al}_{2} \mathrm{O}_{3}$ & 79 & $(-)$ \\
$\mathrm{MgCa}\left(\mathrm{CO}_{3}\right)_{2}-\mathrm{ZrSiO}_{4}$ & 72 & 49 \\
\hline
\end{tabular}

Nota: (-) indica que el calculo no pudo ser realizado debido a la gran dispersión en los datos registrados para esas fases.

culados en el presente trabajo para el $\mathrm{MgCa}\left(\mathrm{CO}_{3}\right)_{2}$ y el $\mathrm{CaCO}_{3}$ en la dolomita y en las composiciones estudiadas.

Igualmente, en la muestra de dolomita pura, se determinó mediante refinamiento Rietveld de los difractogramas registrados, la variación con la temperatura de los parámetros cristalinos $a$ y $c$ de la celda unidad del $\mathrm{MgCa}\left(\mathrm{CO}_{3}\right)_{2}$. La dependencia de estos parámetros con la temperatura entre $25^{\circ}$ y $700^{\circ} \mathrm{C}$ se ajustó a las siguientes expresiones:

$$
\begin{aligned}
& \alpha_{a}\left(20^{\circ}-700^{\circ} \mathrm{C}\right)=4,8+1,277 \cdot 10^{-5} \mathrm{~T}+1,8355 \cdot 10^{-8} \mathrm{~T}^{2} \\
& \alpha_{c}\left(20^{\circ}-600^{\circ} \mathrm{C}\right)=16,066+3,3099 \cdot 10^{-4} \mathrm{~T}+1,3653 \cdot 10^{-7} \mathrm{~T}^{2}
\end{aligned}
$$




\section{CONCLUSIONES}

Se ha puesto en evidencia que la termodifractometría de neutrones es una técnica idónea para el estudio in situ de cinéticas de descomposición y procesos de sinterización reactiva en condiciones no-isotermas.

Como consecuencia de los estudios realizados, con una resolución en temperatura de $5^{\circ} \mathrm{C}$, se ha determinado que:

El proceso de descomposición del $\mathrm{MgCa}\left(\mathrm{CO}_{3}\right)_{2}$ tiene lugar en dos etapas sucesivas: a) descomposición del $\mathrm{MgCa}\left(\mathrm{CO}_{3}\right)_{2}$ generando $\mathrm{CaCO}_{3}$ y $\mathrm{MgO}$, b) descomposición del $\mathrm{CaCO}_{3}$.

Durante el proceso de reacción del $\mathrm{CaO}$ y el $\mathrm{MgO}$ con el $\mathrm{Al}_{2} \mathrm{O}_{3}$ no se ha detectado la formación de fases transitorias. La secuencia de formación de las distintas fases estables $\left(\mathrm{CaAl}_{2} \mathrm{O}_{4^{\prime}}, \mathrm{MgAl}_{2} \mathrm{O}_{4}\right.$ y CaAl $\left.\mathrm{O}_{7}\right)$ concuerda con sus energías libres de formación.

El proceso de reacción de los productos de descomposición del $\mathrm{MgCa}\left(\mathrm{CO}_{3}\right)_{2}$ con el $\mathrm{ZrSiO}_{4}$ tiene lugar con la formación de fases transitorias tales como $\mathrm{ZrO}_{2}$-Tetragonal, $\alpha-\mathrm{Ca}_{2} \mathrm{SiO}_{4}$ y $\mathrm{Ca}_{3} \mathrm{MgSi}_{2} \mathrm{O}_{8}$ que conducen a la formación de las fases estables: $\mathrm{MgO}, \mathrm{CaZrO}_{3}$ y Ca $\mathrm{CaO}_{4}$.

Se han determinado las energías de activación correspondientes a la descomposición del $\mathrm{MgCa}\left(\mathrm{CO}_{3}\right)_{2}\left(56-79 \mathrm{Kcal} \mathrm{mol}^{-1}\right)$ y del $\mathrm{CaCO}_{3}$ $\left(49 \mathrm{Kcal} \mathrm{mol}^{-1}\right)$ en compactos de $\mathrm{MgCa}\left(\mathrm{CO}_{3}\right)_{2}$ puro y en las dos mezclas estudiadas.

De la variación con la temperatura de los parámetros de celdilla a y $c$ del $\mathrm{MgCa}\left(\mathrm{CO}_{3}\right)_{2}$ hasta temperaturas próximas a su descomposición se han deducido las siguientes expresiones: $\alpha_{\mathrm{a}}\left(20^{\circ}-700^{\circ} \mathrm{C}\right)=4,8$ $+1,277 \cdot 10^{-5} \mathrm{~T}+1,8355 \cdot 10^{-8} \mathrm{~T}^{2}$ y $\alpha_{c}\left(20^{\circ}-600^{\circ} \mathrm{C}\right)=16,066+3,3099 \cdot 10^{-4} \mathrm{~T}+$ $1,3653 \cdot 10^{-7} \mathrm{~T}^{2}$.

\section{AGRADECIMIENTOS}

Los autores quieren mostrar su agradecimiento al MCyT por la dotación económica recibida: CICYT MAT-2000-0941. La realización de este trabajo ha sido posible gracias al tiempo de medida concedido por el CRG Hispano-Francés del Instituto Laue-Langevin (ILL) de
Grenoble (Francia): experimento CRG-419 y por el comité científico del ILL: experimento 5-25-25.

Así mismo, los autores quisieran mostrar su agradecimiento a: Dr. M. A. Rodríguez, Dra. A. Hidalgo, Dr. J. Campo, Dr. P. Convert y Dr. T. Hansen por su asistencia y asesoramiento durante la realización de los experimentos.

\section{BIBLIOGRAFIA}

1. A. H. De Aza, P. Pena, M. A. Rodríguez, R. Torrecillas \& S. De Aza. “New spinel-containing refractory cements". J. Europ. Ceram. Soc. 23 (2003) 737 $-744)$

2. J. L. Rodríguez, M. A. Rodríguez, S. De Aza, P. Pena. “Reaction sintering of zircon-dolomite mixtures". J. Europ. Ceram. Soc. 21, 3, 343-354, (2001).

3. A. H. De Aza, J. L. Rodríguez, P. Pena. "Corrosion of magnesia-calcium zirconate-based materials by cement clinker". International Workshop on Ceramic \& Metal Interfaces. June 23-27, Oviedo, Spain. (2002).

4. J. L. Rodríguez, A. H. De Aza, P. Pena, J. Campo, P. Convert \& X. Turrillas. "Study of zircon-dolomite reactions monitored by neutron termodifractometry". J. Solid State Chemistry. 166, 426-433 (2002).

5. J. Pannetier. "Time-resolved scattering experiments" en Neutron and synchrotron radiation for condensed matter studies. HERCULES (High European Research Course for Users of Large Experimental Systems). Volumen I. Editado por Springer-Verlag-Les Editions de Physique (France). Vol. I. 425-436 (1994).

6. A. H. De Aza, M. A. Rodriguez, S. De Aza, P. Pena, P. Convert, T. Hansen and $\mathrm{X}$. Turrillas, "Decomposition of dolomite monitored by neutron thermodiffractometry", J. Am. Ceram. Soc., 85 [4] 881-88 (2002)

7. A. H. De Aza, J. L. Rodriguez, P. Pena, X. Turrillas, "In Situ monitoring reaction sintering of dolomite - based systems" Key Engineering Materials. Vols. 206-213, p.p.437-440, (2002)

8. A. H. De Aza, M. Moset, P. Pena, "Materiales de $\mathrm{Al}_{2} \mathrm{O}_{3}-\mathrm{MgAl}_{2} \mathrm{O}_{4}-\mathrm{CaAl}_{12} \mathrm{O}_{19}$ - $\mathrm{Ca}_{2} \mathrm{Mg}_{2} \mathrm{Al}_{28} \mathrm{O}_{46}$ ", Bol. Soc. Esp. Ceram. V., 41 [4] 361-363 (2002).

9. A. W. Coats and J. P. Redfern. "Kinetic parameters from thermogravimetric data", Nature (London), 201 [1] 23-27 (1978)

10. I. Carrizosa, J. M. Criado, F. G. García y M. González. “Influencia de las condiciones experimentales en la Cinética de la Reacción de Descomposición Térmica del $\mathrm{CaCO}_{3}{ }^{\prime \prime}$ Bol. Soc. Esp. Ceram. Vidrio 17 [1] 23-27 (1978).

Recibido: 01.02 .03

Aceptado: 30.11 .03 